\title{
Transgenic primate models inch forward
}

\author{
The recent announcement of a primate transgenic model of Huntington's disease might appear to represent only a \\ limited advance, but given the work's potential, it would be shortsighted to close the door on this line of research.
}

$\Delta \mathrm{r}_{\mathrm{p}}^{\mathrm{r}}$ recent article in Nature reports the first instance of a disease gene being introduced into the embryo of a non-human primate $^{1}$. On the face of it, however, the finding does not seem particularly noteworthy. Three of the five monkeys who carried several copies of the gene for Huntington's disease died soon after birth and their brains showed limited evidence of the pathology typically associated with the disease. The two surviving monkeys each had only one copy of the gene, and of these two, only one showed (mild) symptoms. Longitudinal studies of their development, required to determine how closely this model matches the progression of Huntington's disease in humans, are not yet done. Yet, despite these problems, this research represents an important step in the right direction.

There remain considerable technical challenges before further progress on these primate models can be made. Transgenic approaches typically have a very low yield. Even in rodents, the success rate is typically ten percent of the gene-injected oocytes. Working with monkeys, for which these techniques have not yet been optimized, makes this even more of a challenge. The five precious monkeys described in the Nature report were the result of 130 mature oocytes that were injected with lentiviruses containing the HTT gene and green fluorescent protein, a success rate of less than four percent. Amplifying these problems, primates have only one breeding season a year and give birth to fewer progeny than rodents.

Combining low-yield transgenic techniques with the expense of maintaining a primate colony results in a very resource-intensive research endeavor. It requires not just seed money, but also a steady stream of funding to preserve technical expertise and animal lines. In the current economic climate, such a high level of funding seems tenuous, particularly as much of this work relies heavily on government funding.

Research with primates is always ethically fraught, particularly when invasive techniques are used. The quantity of animals required for transgenic approaches is well beyond the population sizes involved in other kinds of basic primate research, such as physiological or pharmacological studies. It is therefore critical to evaluate whether transgenic primates represent an appropriate use of such a large number of animals.

In the face of these hurdles, it would be easy to suggest that further work on transgenic primates is not worth supporting, but this argument would be extremely short-sighted. There are limitations to our current approaches to modeling human disease and unique benefits to using the rhesus macaque, our close and exhaustively studied relative. These benefits could quite conceivably outweigh the substantial drawbacks.

In the last 15 years, there has been an explosion in the creation of transgenic rodent models of human disease. Analysis of these animals has led to a much greater understanding of the molecularand cellular-level pathologies associated with neurodegenerative diseases such as Alzheimer's, Parkinson's and Huntington's. However, even the best rodent models of movement disorders do not do a good job of replicating the motor and cognitive symptoms typically seen in human patients. These are critical aspects for characterizing disorders, as well as for testing the effects of treatments, and given the greater similarity between primate and human physiology, there is hope that monkeys may provide a more analogous model. For psychiatric disorders such as schizophrenia and autism, the cognitive and behavioral symptoms are central to the disease. It has been nearly impossible to design models in which rodent behavior could be compared to the complex social and affective makeup of humans. In contrast, the social and affective components of rhesus macaque behavior are widely studied and more comparable to that of humans. In recent years, for example, a number of decision-making models have been developed for studying the neural circuitry of reward, motivation and social context in rhesus monkeys. Combining these procedures with functional magnetic resonance imaging or singleunit physiology could provide powerful probes for revealing changes associated with neurological disorders.

Transgenic primate models also have the potential to nicely complement nontransgenic studies in monkeys. For example, injections of the gene for Huntington's directly into the striatum (where primary disease related pathology occurs) have been used to observe the progression of cellular pathology and its relationship to behavioral changes ${ }^{2}$. Chemical lesions in monkeys have also provided some insight into human disorders. 1-methyl 4-phenyl 1,2,3,6-tetrahydropyridine (MPTP) damage to midbrain dopamine neurons (in both human and non human primates) revolutionized the understanding and treatment of Parkinson's disease. However, none of these models can provide insight into the role of genetic factors in the development of disease; genetic manipulations or transgenic models are the only way to study this.

A wide phylogenetic range of transgenic disease models (from worms to mice to primates) can provide much insight into mechanisms of human disease. Given the technical difficulty, as well as the financial and ethical costs associated with transgenic primate models of disease, we must embark on their use with special care. However, this approach offers a unique opportunity to examine the cognitive and affective components of various neurological and neurodegenerative disorders. These five monkeys therefore represent an important proof of principle. They are the seeds of a host of future possibilities and it is critical that we not close the door on this line of research prematurely.

1. Yang, S.-H. et al. Nature advance online publication, 10.1038/nature06975 (18 May 2008).

2. Palfi, S. et al. Mol. Ther. 15, 1444-1451 (2007). 